\title{
THE GENERA OF EDGE AMALGAMATIONS OF COMPLETE BIGRAPHS(1)
}

BY

\section{SETH R. ALPERT}

ABSTRACT. If $G$ and $H$ are graphs, then $G \vee H$ is defined to be a graph obtained by identifying some edge of $G$ with some edge of $H$. It is shown that for all $m, n, p$, and $q$ the genus $g\left(K_{m, n} \vee K_{p, q}\right)$ is either $g\left(K_{m, n}\right)+g\left(K_{p, q}\right)$ or else $g\left(K_{m, n}\right)+g\left(K_{p, q}\right)-1$. The latter value is attained if and only if both $K_{m, n}$ and $K_{p, q}$ are critical in the sense that the deletion of any edge results in a graph whose genus is one less than the genus of the original graph.

I. Throughout this paper graphs will be finite simplicial 1-complexes. The genus $g(G)$ of a connected graph $G$ is the minimum genus of any closed orientable 2-manifold in which $G$ can be imbedded.

If $T$ is a subgraph both of $G$ and of $H$, then a new graph $G \vee_{T} H$ may be formed by identifying a copy of $T$ contained in $G$ with a copy of $T$ contained in $H$. This new graph is called an amalgamation of $G$ and $H$ along $T$, and in general depends on the choice of copies of $T$.

The aim of this paper is to determine the genera of all graphs of the form $K_{m, n} V_{K_{2}} K_{p, q}$. This is achieved in Theorem I.3. Note that, due to the symmetry of complete bigraphs, the amalgamation $K_{m, n} V_{K_{2}} K_{p, q}$ is independent of which edges $K_{2}$ one amalgamates along. To simplify notation, an amalgamation $G \vee_{K_{2}} H$ shall be written simply as $G \vee H$.

Using different methods, Ringel [3] and Schanuel [4] have determined the genera of all complete bigraphs, as follows.

Theorem I. 1. For all integers $m$ and $n$ greater than or equal to 2,

$$
g\left(K_{m, n}\right)=\{(m-2)(n-2) ; 4\}
$$

where $\{r\}$ denotes the least integer greater than or equal to any real number $r$.

Received by the editors December 21, 1972.

AMS (MOS) subject classifications (1970). Primary 05C10; Secondary 55A15.

Key words and phrases. Genus, Euler formula, bigraph, orientable 2-manifold.

(1) This research was conducted while the author was a graduate student at Columbia University. He wishes to thank Professors Hyman Bass and Jonathan Gross for their advice and support during that period. 
A graph $G$ is said to be critical if, for each edge $e$ of $G, g(G \sim e)=g(G)-1$. Ringel's proof of Theorem I.1 also yields a determination of which complete bigraphs are critical.

Theorem I.2. The complete bigraph $K_{m, n}$ is critical if and only if $m$ and $n$ are greater than 1 and either $m \equiv n \equiv 1(\bmod 4)$ or $m \equiv n \equiv 3(\bmod 4)$.

The major result of this paper is the following theorem.

Theorem I.3. (1) For all positive integers $m, n, p$, and $q, g\left(K_{m, n} \vee K_{p, q}\right)$ is either $g\left(K_{m, n}\right)+g\left(K_{p, q}\right)$ or $g\left(K_{m, n}\right)+g\left(K_{p, q}\right)-1$.

(2) $g\left(K_{m, n} \vee K_{p, q}\right)=g\left(K_{m, n}\right)+g\left(K_{p, q}\right)-1$ if and only if botb $K_{m, n}$ and $K_{p, q}$ are critical.

Note that in combination with Theorems I.1 and I.2, this yields a determination of the genera of all amalgamations of complete bigraphs along an edge.

In $\S$ II the machinery to be used in the proof of Theorem I. 3 is set up and part (1) is established. The fact that $g\left(K_{m, n} \vee K_{p, q}\right)=g\left(K_{m, n}\right)+g\left(K_{p, q}\right)-1$ implies that $K_{m, n}$ and $K_{p, q}$ are critical is demonstrated in $\S$ III and the converse proposition is proven in $\S I V$.

Similar results determining the genera of graphs of the form $K_{m} V_{K_{p}} K_{n}, 2 \leq$ $p \leq 5$, in many cases, have been obtained in Alpert [1].

II. This section is devoted to setting up the machinery to be used in the proof of Theorem I.3 and to proving its first assertion.

If $f: G \rightarrow M$ is an imbedding of a graph $G$ in a surface $M$ then the image of $f$ is also called $G$, and the components of $M \sim G$ are called faces of the imbedding. If all the faces are open 2-cells the imbedding is called a 2-cell imbedding. It is well known that any imbedding of a graph in a surface of minimal genus has this property. Of course, the Euler formula applies to any 2-cell imbedding.

The boundary of any face of a 2-cell imbedding of a graph $G$ is a closed walk of $G$. It is most convenient to refer to this closed walk rather than the face itself. Its edges will be called sides of the face.

Because all closed walks of a bigraph are of even length, any face of a 2-cell imbedding of a bigraph must have an even number of sides, and this number must be at least 4. This observation leads to the following definition, in analogy to the triangulation deficiency used in Alpert [1].

The quadrilateral deficiency $q(G ; g)$ of a bigraph $G$ for a genus $g$ is defined by the equation

$$
q(G ; g)=8 g-8+4 V(G)-2 E(G)
$$

In case $g=g(G)$, one simply refers to the quadrilateral deficiency of $G$ and writes $q(G)$ instead of $q(G ; g(G))$. 
If there is a 2 -cell imbedding of $G$ in a surface of genus $g$ with $F$ faces, then an elementary manipulation of the Euler formula shows that $q(G ; g)=2 E(G)$ $-4 F$.

The following proposition offers some clarification of this terminology.

Proposition II.l. Let $G$ be a bigraph.

(1) If there is a 2-cell imbedding of $G$ in a surface of genus $g$ and if $F_{k}$ is the number of $k$-sided faces, then

$$
q(G ; g)=\sum_{k \geq 4}(k-4) F_{k} .
$$

(2) If $q(G ; g) \geq 0$, then $1 / 2 q(G ; g)$ edges must be added to a 2-cell imbedding of $G$ in a surface of genus $g$ in order to make all faces quadrilaterals.

(3) If $q(G ; g)<0$, then $G$ cannot be imbedded in a surface of genus $g$, and at least $1 / 2|q(G ; g)|$ edges must be removed from $G$ in order to make such an imbedding possible (although this may not always work).

Proof. (1) Note that in any 2-cell imbedding of $G, 2 E(G)=\Sigma k F_{k}$. Thus

$$
q(G ; g)=2 E(G)-4 F=\sum k F_{k}-4 \sum F_{k}=\sum(k-4) F_{k} \text {. }
$$

Moreover, because $G$ is a bigraph there are no faces with fewer than 4 sides, so the sum may be taken over all $k \geq 4$.

(2) By (1), if there exists a 2-cell imbedding of $G$ in a surface of genus $g$, then $q(G ; g)=\Sigma_{k \geq 4}(k-4) F_{k}$. Hence if $q(G ; g)>0$, there is at least one $n$-sided face $P$ with $n \geq 6$. A new edge (possibly multiple) can be drawn across $P$ so as to subdivide it into a 4 -sided face and an $(n-2)$-sided face. Clearly, if the resulting graph is $G^{\prime}$, then $q\left(G^{\prime} ; g\right)=q(G ; g)-2$. Iteration of this procedure gives the desired result. (Of course, $q(G ; g) \geq 0$ does not guarantee the existence of an imbedding of $G$ in a surface of genus $g$.)

(3) By (1), if $q(G ; g)<0$, there is no 2 -cell imbedding, and so no imbedding at all, of $G$ in a surface of genus $g$. If $1 / 2|q(G ; g)|$ edges are removed from $G$, the resulting graph $G^{\prime}$ has $q\left(G^{\prime} ; g\right)=0$. Thus it may be possible to imbed $G^{\prime}$ in a surface of genus $g$.

As a corollary to Proposition II.1, one obtains a standard lower bound for the genus of an arbitrary bigraph $G$.

Corollary II.2. For any bigraph $G, g(G) \geq\{E(G) / 4-V(G) / 2+1\}$.

Proof. By definition of $q(G)$ and by Proposition II.1 $q(G) \geq 0$. That is, $8 g(G)-8+4 V(G)-2 E(G) \geq 0$. The conclusion is an easy consequence of the above inequality.

Proposition II.3. For all bigraphs $G, H$, and $T$,

$$
q\left(G \vee_{T} H ; g(G)+g(H)-g(T)+x\right)=q(G)+q(H)-q(T)+8 x .
$$


Consequently,

$$
g\left(G \quad v_{T} H\right) \geq g(G)+g(H)+g(T)+\{(q(T)-q(G)-q(H)) / 8\} .
$$

Proof. Obviously, $V\left(G \quad V_{T} H\right)=V(G)+V(H)-V(T)$ and $E\left(G \quad V_{T} H\right)=E(G)+$ $E(H)-E(T)$. Thus the first equation is an immediate consequence of the defining equation for $q(G, g)$.

As for the second equation, observe that there exists an integer $x$ such that

$$
g\left(G \quad T^{H}\right)=g(G)+g(H)-g(T)+x .
$$

Since $g\left(G \vee_{T} H\right) \geq 0$, it follows from the first equation that

$$
x \geq\{(q(T)-q(G)-q(H)) / 8\},
$$

which completes the proof.

Proposition II.4. For all $m, n \geq 2$,

$$
q\left(K_{m, n}\right)=8(\{(m-2)(n-2) / 4\}-(m-2)(n-2) / 4) .
$$

Proof. By Theorem I.1 and definition of $q(G)$,

$$
\begin{aligned}
q\left(K_{m, n}\right) & =8\{(m-2)(n-2) / 4\}-8+4 m+4 n-2 m n \\
& =8(\{(m-2)(n-2) / 4\}-(m-2)(n-2) / 4) .
\end{aligned}
$$

Using the formula obtained in Proposition II.4 one may express $q\left(K_{m, n}\right)$ as a function of the residue classes of $m$ and $n$ modulo 4. These values are tabulated in Table 1.

\section{TABLE 1}

\begin{tabular}{c|c|c|c|c|}
$q\left(K_{m, n}\right)$ & as a function of $m$ and $n \bmod$ \\
\hline 0 & 0 & 4 & 0 & 4 \\
\hline 1 & 4 & 6 & 0 & 2 \\
\hline 2 & 0 & 0 & 0 & 0 \\
\hline 3 & 4 & 2 & 0 & 6 \\
\hline
\end{tabular}

Observe that $q\left(K_{m, n}\right)$ attains exactly 4 different values. Using the formula obtained in Proposition II.3, one may compute the value of $q\left(K_{m, n} \vee K_{p, q} ; g\left(K_{m, n}\right)+g\left(K_{p, q}\right)-1\right)$ as a function of $q\left(K_{m, n}\right)$ and $q\left(K_{p, q}\right)$, as is shown in Table 2. 
TABLE 2

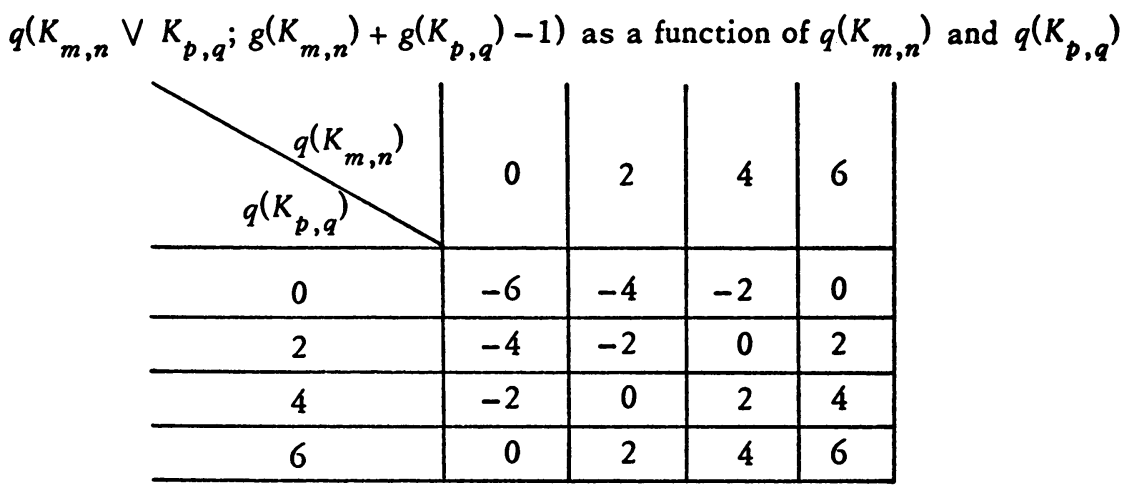

Note that for an arbitrary edge amalgamation $G \vee H$, the genus is at most the sum of the genera of $G$ and $H$. To see this, take minimal imbeddings of $G$ and $H$ in surfaces $M$ and $N$, respectively. The edge $e$ along which $G$ and $H$ are to be amalgamated must be the side of some face $P$ of the imbedding of $G$ and also some face $P^{\prime}$ of the imbedding of $H$. If an open disk whose boundary intersects the boundary of $P$ only in $e$ is excised from $P$ and a similar disk is excised from $P^{\prime}$, one may form the connected sum of $M$ and $N$ by identifying the boundaries in such a way so that the 2 copies of $e$ match up. This gives an imbedding of $G \vee H$ in a surface of genus $g(G)+g(H)$, thereby proving

Proposition II.5. For all graphs $G$ and $H, g(G \vee ' H) \leq g(G)+g(H)$.

Now observe that a consequence of Table 2 and Propositions II. 1 and II. 3 is that for all $m, n, p$, and $q$,

$$
g\left(K_{m, n} \vee K_{p, q}\right) \geq g\left(K_{m, n}\right)+g\left(K_{p, q}\right)-1 .
$$

Hence in any case, $g\left(K_{m, n} \vee K_{p, q}\right)$ is either $g\left(K_{m, n}\right)+g\left(K_{p, q}\right)$ or $g\left(K_{m, n}\right)+$ $g\left(K_{p, q}\right)-1$, which establishes (1) of Theorem I.3.

III. The rest of the proof of Theorem I.3 is by cases according to the value of $q\left(K_{m, n} \vee K_{p, q} ; g\left(K_{m, n}\right)+g\left(K_{p, q}\right)-1\right)$. By Proposition II.1, if $q(G ; g)<0$, then $G$ cannot be imbedded in a surface of genus $g$. Moreover, it follows from Theorem I.2 and Table 1 that $K_{m, n}$ is critical if and only if $q\left(K_{m, n}\right)=6$. Now consulting Table 2, one sees that in order to prove (2) of Theorem I.3, it suffices to prove the following:

(1) $g\left(K_{m, n} \vee K_{p, q}\right)=g\left(K_{m, n}\right)+g\left(K_{p, q}\right)-1$ implies that

$$
q\left(K_{m, n} \vee K_{p, q} ; g\left(K_{m, n}\right)+g\left(K_{p, q}\right)-1\right)=6 .
$$

(2) If $K_{m, n}$ and $K_{p, q}$ are critical, then 


$$
g\left(K_{m, n} \vee K_{p, q}\right)=g\left(K_{m, n}\right)+g\left(K_{p, q}\right)-1 \text {. }
$$

In this section (1) will be proven by showing that $K_{m, n} \vee K_{p, q}$ cannot be imbedded in a surface of genus $g\left(K_{m, n}\right)+g\left(K_{p, q}\right)-1$ when $q\left(K_{m, n} \vee K_{p, q}^{m, n} ; g\left(K_{m, n}\right)+g\left(K_{p, q}\right)-1\right)<6$. The proof of Theorem I. 3 is completed in the next section by constructing an imbedding of $K_{m, n} \vee K_{p, q}$ in a surface of genus $g\left(K_{m, n}\right)+g\left(K_{p, q}\right)-1$ in the case when $K_{m, n}$ and $K_{p, q}$ are both critical.

Before proceeding, it is necessary to recall some basic terminology about imbeddings of graphs in orientable surfaces. For details, see Alpert [1].

Let $x$ be a vertex of a graph $G$ imbedded in a surface $M$. The orientation on $M$ induces a cyclic ordering of the vertices adjacent to $x$ called the adjacency tour at $x$ and written in the form $x) a, b, c, \cdots, d, e$. Recall that an imbedding of $G$ in an orientable surface is equivalent to a list of adjacency tours. It is assumed that the reader is familiar with this equivalence, which is explained in $\S_{1}$ of Alpert [1].

Faces of an imbedding of a graph in an orientable surface are denoted by the cyclic sequence of vertices of the face written in the reverse order to that induced by the orientation and enclosed by square brackets. If $P=[a, b, c, \ldots]$ is a face of some imbedding, then $a b c$ is called an angle of $P$ with endpoints $a$ and $c$ and center $b$.

Note that the vertices of $G \vee H=G V_{K_{2}} H$ are naturally partitioned into the sets $G \sim K_{2}, H \sim K_{2}$ and $K_{2}$. Standard procedure here will be to denote the vertices of $G \sim K_{2}$ by $g_{1}, g_{2}, \ldots$, those of $H \sim K_{2}$ by $b_{1}, b_{2}, \ldots$ and those of $K_{2}$ by $x$ and $y$. An amalgamating angle of an imbedding of $G \vee H$ in an orientable surface is defined to be an angle with center $x$ or $y$ and one endpoint from $G \sim K_{2}$ and the other from $H \sim K_{2}$. When writing the adjacency tours at vertices of $G \vee H$, a sequence $g_{1}, \cdots, g_{2}$ or $b_{1}, \cdots, b_{2}$ will consist entirely of vertices of $G \sim K_{2}$ or $H \sim K_{2}$, respectively. The possibility that the sequence $g_{1}, \cdots, g_{2}$ is of length 1 is allowed.

Using these conventions note that any juxtaposition of a $g_{i}$ and $b_{j}$ in the adjacency tour at $x$ or $y$ yields an amalgamating angle on some face $P$ of the imbedding. Because no $g_{i}$ is adjacent to an $b_{j}$, there must be at least one more occurrence of a vertex of $K_{2}$ on $P$. Moreover, if $G$ and $H$ are bipartite, then because $x$ and $y$ are adjacent in $G$ and $H$, it follows that $P$ must be at least 6-sided. Since there must be at least one juxtaposition of a $g_{i}$ and an $b_{j}$ in the adjacency tour at $x$ for any imbedding of $G \vee H$, it follows that $q(G \vee H) \geq 2$ for all bipartite $G$ and $H$. This proves the following

Proposition III.1. If $G \vee H$ can be imbedded in a surface of genus $g$, then $q(G \vee H ; g) \geq 2$. 
Theorem III.2. (1) If $G \vee H$ can be imbedded in a surface of genus $g(G)+$ $g(H)-1$, then each vertex of $K_{2}$ is the center of at least two amalgamating angles.

(2) Consequently, $q(G \vee H ; g(G)+g(H)-1) \geq 4$.

Proof. (1) Let $x$ be a vertex of $K_{2}$. Because there must be at least one juxtaposition of a $g_{i}$ with an $b_{j}$ in the adjacency tour at $x, x$ is the center of at least one amalgamating angle. If $x$ is the center of exactly one amalgamating angle, then the adjacency tour at $x$ has the form $x) y, g_{1}, \cdots, g_{2}, b_{2}, \ldots, b_{1}$ where $y$ is the other vertex of $K_{2}$. It is now possible to "split" the vertex $x$ in such a way so as to obtain an imbedding of $G V_{K_{1}} H$ in the same surface. To do this, replace $x$ by $x_{1}$ in every adjacency tour at a $g_{i}$, replace $x$ by $x_{2}$ in every adjacency tour at an $b_{j}$, and replace the adjacency tour at $x$ by the adjacency tours

$$
\left.\left.x_{1}\right) y, g_{1}, \cdots, g_{2}, x_{2}\right) y, b_{2}, \cdots, b_{1} \text {. }
$$

Finally, in the adjacency tour at $y$, replace $x$ by $x_{1}, x_{2}$.

This clearly gives a scheme for $G V_{K_{1}} H$. The only change in faces from the original imbedding is that the face $P$ with amalgamating angle $g_{2} x h_{2}$ is expanded from

$$
P=\left[g_{2}, x, b_{2} \cdots\right] \text { to } P=\left[g_{2}, x_{1}, y, x_{2}, b_{2}, \ldots\right] \text {. }
$$

Thus the number of faces is the same as in the original imbedding. Moreover, the numbers of edges and vertices have each increased by 1 , so the Euler characteristic is unaffected by these changes. This means that $G V_{K_{1}} H$ is imbedded in a surface of genus $g(G)+g(H)-1$ contradicting a theorem of Battle, Harary, Kodama, and Youngs [2].

Hence $x$ is the center of at least 2 amalgamating angles.

(2) An amalgamating angle must lie on a face with 6 or more sides. Moreover, it is impossible to have a repeated vertex on a 6 -sided face of an imbedding of a bigraph. Hence $x$ lies on at least 2 faces with 6 sides or else on at least one face with 8 or more sides. In either case it must be true that $q(G \vee H ; g(G)+g(H)-1) \geq 4$.

Theorem III.3. If $G \vee H$ can be imbedded in a surface of genus $g(G)+g(H)-1$, then $q(G \vee H ; g(G)+g(H)-1) \geq 6$.

Proof. Suppose $G \vee H$ is embedded in a surface of genus $g(G)+g(H)-1$. The previous theorem shows that $q(G \vee H ; g(G)+g(H)-1) \geq 4$ and that if $q(G \vee H ; g(G)+g(H)-1)=4$, then one of the vertices $x$ of $K_{2}$ must lie on exactly two amalgamating angles. The adjacency tours at $x$ and $y$ therefore look like

$$
\begin{aligned}
& x) y, g_{1}, \cdots, g_{2}, b_{2}, \cdots, b_{3}, g_{3}, \ldots \\
& \text { y) } x, g_{6}, \cdots, g_{5}, b_{5}, \cdots, b_{4}, g_{4}, \ldots .
\end{aligned}
$$


Once again, the procedure is to "split" $x$ so as to obtain an imbedding of $G V_{K_{1}} H$ in the same surface, contradicting the theorem of Battle, Harary, Kodama, and Youngs [2].

Because $g(G \vee H ; g(G)+g(H)-1)=4$ and since no $g_{i}$ is adjacent to an $b_{j}$, the only faces with more than 4 sides are hexagons

$$
P_{1}=\left[g_{2}, x, b_{2}, b_{4}, y, g_{4}\right] \text { and } P_{2}=\left[h_{3}, x, g_{3}, g_{5}, y, b_{5}\right] \text {. }
$$

Now we split $x$ into 2 new vertices as follows:

In the adjacency tours at the $g_{i}$ 's change $x$ to $x_{1}$, in those at the $b_{j}$ 's change $x$ to $x_{2}$, in the adjacency tour at $y$, replace $x$ by $x_{1}$ and insert an $x_{2}$ following $b_{4}$, thus

$$
\text { y) } x_{1}, g_{6}, \cdots, g_{5}, b_{5}, \ldots, b_{4}, x_{2}, g_{4}, \ldots
$$

Finally, replace the adjacency tour at $x$ by adjacency tours

$$
\left.\left.x_{1}\right) y, g_{1}, \ldots, g_{2}, g_{3}, \ldots x_{2}\right) y, b_{2}, \ldots, b_{3} .
$$

The only change in faces from the original imbedding is that $P_{1}$ and $P_{2}$ are now consolidated into new faces

$$
P_{1}^{\prime}=\left[g_{2}, x_{1}, g_{3}, g_{5}, y, b_{5}, b_{3}, x_{2}, y, g_{4}\right] \text { and } P_{2}^{\prime}=\left[y, x_{2}, b_{2}, b_{4}\right] \text {. }
$$

Hence the Euler characteristic is unchanged and so the new scheme is for an imbedding of $G \vee_{K_{1}} H$ in a surface of genus $g(G)+g(H)-1$, a contradiction. Hence $q(G \vee H ; g(G)+g(H)-1) \geq 6$.

Corollary III.4. If $g\left(K_{m, n} \vee K_{p, q}\right)=g\left(K_{m, n}\right)+g\left(K_{p, q}\right)-1$, then $q\left(K_{m, n} \vee K_{p, q} ; g\left(K_{m, n}\right)+g\left(K_{p, q}\right)-1\right)=6$.

Proof. This is an immediate consequence of Theorem III.3 and Table 2.

IV. The proof of Theorem I.3 is completed by the following theorem.

Theorem IV.1. If $K_{m, n}$ and $K_{p, q}$ are critical then $g\left(K_{m, n} \vee K_{p, q}\right)=$ $g\left(K_{m, n}\right)+g\left(K_{p, q}\right)-1$.

Proof. The theorem will be proved once an imbedding of $K_{m, n} \vee K_{p, q}$ in a surface of genus $g\left(K_{m, n}\right)+g\left(K_{p, q}\right)-1$ has been constructed.

First note that if $K_{m, n}$ is critical then there is a minimal imbedding of $K_{m, n}$ for which the only face with more than four sides is 10 -sided of the form $[a, x, c$, $w, b, x, a, z, c, y]$. To see this, observe that $m, n \geq 3$ and that if $K_{2}=(a, x)$, $K_{m, n} \sim K_{2}$ can be quadrilaterally imbedded in a surface of genus $g\left(K_{m, n}\right)-1$. Assume that one face of this imbedding is $[a, z, c, y]$. Then because $a$ and $x$ are adjacent in $K_{m, n}, x$ must be adjacent to $c$ and so there is another face of the form $[x, c, w, b]$ (if $m=n=3$, then $w=z$ ). Now excise open disks from the interiors of these 2 faces and identify the boundaries. It is now possible to add the edge 
$(x, a)$ and thereby obtain a minimal imbedding of $K_{m, n}$ of the desired type.

Similarly, there is a minimal imbedding of $K_{p, q}$ for which the only face with more than 4 sides is 10 -sided of the form $\left[x^{\prime}, a^{\prime}, y^{\prime}, c^{\prime}, z^{\prime}, a^{\prime}, x^{\prime}, b^{\prime}, w^{\prime}, c^{\prime}\right]$.

We shall now amalgamate $K_{m, n}$ and $K_{p, q}$ along the edges $(x, c)$ and $\left(x^{\prime}, c^{\prime}\right)$. Note that the adjacency tours at $x, c, x^{\prime}, c^{\prime}$ are as follows:
x) $b, a, c, \cdots$
$\left.x^{\prime}\right) c^{\prime}, a^{\prime}, b^{\prime}, \ldots$
c) $x, w, \cdots, z, y, \ldots$
$\left.c^{\prime}\right) y^{\prime}, z^{\prime}, \ldots, w^{\prime}, x^{\prime}, \ldots$.

Form new adjacency tours

$$
\begin{aligned}
& \left.x^{\prime \prime}\right) c^{\prime \prime}, \ldots, b, a^{\prime}, a, b^{\prime}, \ldots \\
& \left.c^{\prime \prime}\right) x^{\prime \prime}, \ldots, y^{\prime}, w, \ldots, z, z^{\prime}, \ldots, w^{\prime}, y, \ldots .
\end{aligned}
$$

The remaining adjacency tours are appended, with $x$ and $c$ changed to $x^{\prime \prime}$ and $c^{\prime \prime}$, respectively.

If all new juxtapositions of vertices in the new adjacency tours at $x^{\prime \prime}$ and $c^{\prime \prime}$ are accounted for, we will obtain a list of the new faces. They are:

$$
\left[b, x^{\prime \prime}, a^{\prime}, y^{\prime}, c^{\prime \prime}, w\right], \quad\left[a^{\prime}, x^{\prime \prime}, a, z, c^{\prime \prime}, z^{\prime}\right], \quad\left[a, x^{\prime \prime}, b^{\prime}, w^{\prime}, c^{\prime \prime}, y\right] \text {. }
$$

If the imbedding of $K_{m, n}$ has $F_{1}$ faces and that of $K_{p, q}$ has $F_{2}$ faces, then the number of faces in the new imbedding is $F_{1}+F_{2}+1$. The new scheme is for an imbedding of $K_{m, n} \vee K_{p, q}$ and since $V\left(K_{m, n} \vee K_{p, q}\right)=V\left(K_{m, n}\right)+V\left(K_{p, q}\right)-2$ and $E\left(K_{m, n} \vee K_{p, q}\right)=E\left(K_{m, n}\right)+E\left(K_{p, q}\right)-1$, it follows from the Euler formula that the genus $g$ of the new surface is

$$
\begin{aligned}
g & =1-1 / 2\left(V\left(K_{m, n}\right)+V\left(K_{p, q}\right)-2-E\left(K_{m, n}\right)-E\left(K_{p, q}\right)+1+F_{1}+F_{2}+1\right) \\
& =1-1 / 2\left(V\left(K_{m, n}\right)-E\left(K_{m, n}\right)+F_{1}\right)+1-1 / 2\left(V\left(K_{p, q}\right)-E\left(K_{p, q}\right)+F_{2}\right)-1 \\
& =g\left(K_{m, n}\right)+g\left(K_{p, q}\right)-1,
\end{aligned}
$$

as desired.

\section{REFERENCES}

1. S. Alpert, The genera of amalgamations of graphs, Trans. Amer. Math. Soc. 178 (1973), 1-40.

2. J. Battle, F. Harary, Y. Kodama and J. W. T. Youngs, Additivity of the genus of a graph, Bull. Amer. Math. Soc. 68 (1962), 565-568. MR 27 \#5247.

3. G. Ringel, Das Geschlecht des vollständigen paaren Graphen, Abh. Math. Sem. Univ. Hamburg 28 (1965), 139-150. MR 32 \#6439.

4. S. Schanuel, personal communication.

DEPARTMENT OF MATHEMATICS, COLUMBIA UNIVERSITY, NEW YORK, NEW YORK 10027

Current address: State University of New York, Downstate Medical Center, Department of Medical Computer Science, Brooklyn, New York 11203 